\title{
CONTACT PROBLEMS IN INDUSTRIAL APPLICATIONS USING FREEFEM
}

\author{
Houssam Houssein $^{1}$, Simon Garnotel ${ }^{2}$ AND Frédéric Hecht ${ }^{3}$ \\ ${ }^{1}$ Sorbonne Université, Laboratoire Jacques-Louis Lions \\ F-75005 Paris, France \\ houssein@1jll.math.upmc.fr \\ ${ }^{2}$ Airthium SAS \\ Accelair, 1 chemin de la Porte des Loges, 78350, Les Loges-en-Josas, France \\ simon.garnotel@airthium.com \\ ${ }^{3}$ Sorbonne Université, Laboratoire Jacques-Louis Lions \\ F-75005 Paris, France \\ frederic.hecht@sorbonne-universite.fr
}

Key words: Contact Problems, FEM, Interior point method, Penalty method, Symmetric formulation

\begin{abstract}
This paper presents an algorithm and a method to solve mechanical contact problems between two bodies or more, for linear elastic and finite deformation problems. The contact problem is considered as an optimization one, more specifically as a minimization problem. The interior point method is used to solve the minimization problem. This algorithm is symmetric and the user does not need to specify anymore a slave and a master body. The algorithm was developed using FreeFEM and IPOPT software.
\end{abstract}

\section{INTRODUCTION}

The mechanical contact between two bodies is one of the difficult problems in solid mechanics, indeed the contact area is unknown. There exist several algorithms to solve the contact problems $[8,9]$, most of them involve the concept of master/slave, which prevents the penetration of the slave body into the master one, and therefore causes the non-symmetry of the algorithm. Otherwise the interior point method was not widely used in contact problems, we can cite for example [10,11, 12].

In this paper, we restrict ourselves to the contact between two hyperelastic bodies (where the linear elastic body is a particular case). The frictional case is not considered here. One of the goals of this work is to propose a method that uses the FreeFEM [1] software and its tools to solve the contact problem. As it is formulated as an optimization problem, the interior point method is used and the Interior Point OPTimizer algorithm (IPOPT) [5], which is already interfaced with FreeFEM, is used to solve the optimization problem and to reach the solution. The second goal of this work is to obtain a symmetric formulation, in order to allow the user to no longer distinguish between slave and master bodies. An algorithm to solve

This work was partially funded by the "Association Nationale de la Recherche et de la Technologie" (ANRT) 
Signorini's problem using FreeFEM and IPOPT software can be found in [2].

This paper is organized as follows: in section 2, we present briefly the contact problem and the symmetric formulation. In section 3, we present the numerical methods, used to solve the constrained minimization problems and we show that it will not generate numerical difficulties, in addition to the convergence of the method. We also introduce briefly alternatives symmetric formulations of the contact problems in section 4, and we present briefly the contact algorithm in section 5. Finally, in section 6, two examples are studied in order to show that the algorithm can handle large deformations, and multi-body contact in 3D.

\section{Contact Problem and discretization}

In the following we consider the contact between two bodies $\Omega_{1}$ and $\Omega_{2}$, where $\Omega_{i}$ denotes the body $i$ in its reference configuration, for the sake of simplicity we suppose that $\Omega_{i} \subset \mathbb{R}^{2}$, the same work can be done for three-dimensional space. We note $\Gamma_{d}^{i}$ the part of the boundary $\partial \Omega_{i}$ where a displacement is imposed, $\Gamma_{l}^{i} \subset \partial \Omega_{i}$ where the load is imposed, $\Gamma_{c}^{i} \subset \partial \Omega_{i}$ the potential contact part of the boundary $\partial \Omega_{i}$. We also suppose that $\Gamma_{d}^{i}, \Gamma_{l}^{i}, \Gamma_{c}^{i}$ are disjoint and $\partial \Omega_{i}=\Gamma_{d}^{i} \cup \Gamma_{l}^{i} \cup \Gamma_{c}^{i}$ where $\Gamma_{c}^{i}$ has a non null area. Let $\mathbf{X}$ be a material point in $\Omega=\Omega_{1} \cup \Omega_{2}$, then the displacement field $\mathbf{u}$ is defined by $\mathbf{u}=\mathbf{x}-\mathbf{X}$, where $\mathbf{x}=\phi(\mathbf{X})$ is the actual displacement of $\mathbf{X}$, and $\phi$ describes the transformation of the initial or reference configuration $\Omega$ into the actual configuration $\phi(\Omega)$, due let's say to a load applied on $\Omega$.

\section{Let $\mathbf{f}$ be a surface traction applied at $\Gamma_{l}=\Gamma_{l}^{1} \cup \Gamma_{l}^{2}$, and a null displacement imposed at $\Gamma_{d}=\Gamma_{d}^{1} \cup \Gamma_{d}^{2}$, thus}

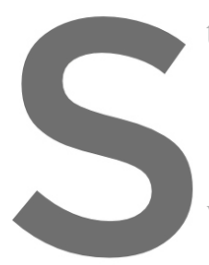

the contact problem can be
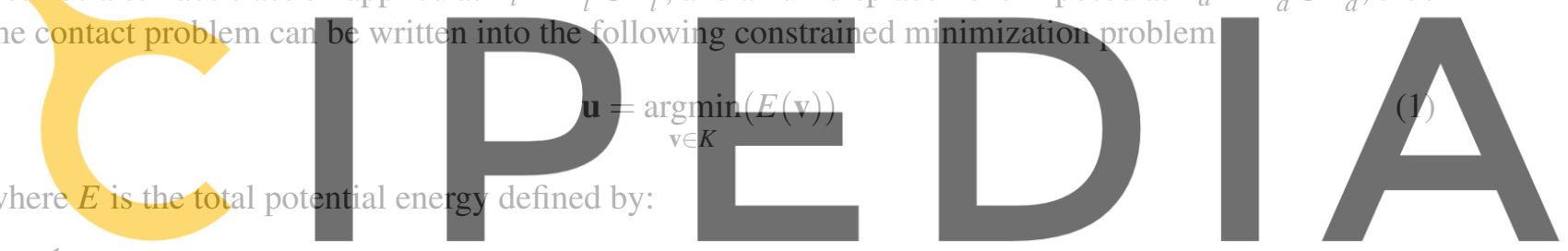

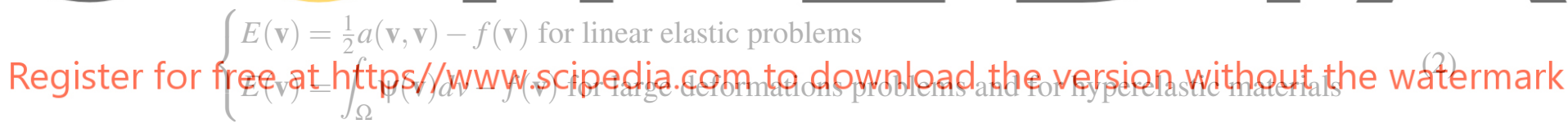

The application $a$ is continuous, coercive, symmetric, bilinear and is defined by $a(\mathbf{u}, \mathbf{v})=\int_{\Omega} C \varepsilon(\mathbf{u})$ : $\varepsilon(\mathbf{v}) d v$, the application $f$ is linear and continuous and is defined by $f(\mathbf{v})=\int_{\Gamma_{l}} \mathbf{f} . \mathbf{v} d s$, the material tensor $C$ and the strain energy function $\psi$ describe the behavior of the material. The set $K$ is defined by:

$$
K=\left\{\mathbf{v} \in\left(H^{1}\left(\Omega_{1}\right)\right)^{2} \times\left(H^{1}\left(\Omega_{2}\right)\right)^{2} ; \mathbf{v}=0 \text { on } \Gamma_{d} ;\left(\mathbf{x}-\overline{\mathbf{x}}_{2}\right) \mathbf{n} \geq 0 \text { on } \Gamma_{c}^{1}\right\}
$$

where $\overline{\mathbf{x}}_{2}$ is the projection of $\mathbf{x}=\mathbf{X}+\mathbf{v} \in \phi\left(\Gamma_{c}^{1}\right)$ on $\phi\left(\Gamma_{c}^{2}\right)$ and $\mathbf{n}$ is the outward unit normal vector at $\overline{\mathbf{x}}_{2}$. The set $K$ describes the non-penetration between the two bodies.

\subsection{Discretization}

The mesh $\Omega_{h}$ is composed from the triangles family $\left\{T_{i} \mid i=1, \ldots, n_{T}\right\}$, we then consider the space $V_{h}=\left\{\mathbf{v}=\left(v_{1}, v_{2}\right) \in C^{1}\left(\Omega_{h}\right) \mid \mathbf{v}_{\mid T_{i}} \in P^{r} \times P^{r}, \forall i=1, \ldots, n_{T}\right.$ and $\mathbf{v}=0$ on $\left.\Gamma_{0}\right\}$ where $r=1$ or 2 . The non- 
penetration constraints are expressed in a weak form as in $[3,4]$. The minimization problem in this case becomes

Find $\mathbf{u} \in V_{h}$ such that

$$
\left\{\begin{array}{l}
\mathbf{u}=\underset{\mathbf{v} \in V_{h}}{\operatorname{argmin}}(E(\mathbf{v})) \\
\int_{\Gamma_{C 1}}\left(\left(\mathbf{x}-\overline{\mathbf{x}}_{2}\right) \mathbf{n}\right) \cdot \phi_{i}^{(1)} d s \geq 0 \quad \forall i=1, \ldots, n_{C 1}
\end{array}\right.
$$

where $n_{C 1}$ is the contact nodes number of $\Gamma_{c}^{1}$ and $\phi_{i}^{(1)}$ is the shape function at each of these latter. In this formulation, we implicitly choose the first body as slave and the second one as master (otherwise speaking the first discretized body $\Omega_{h 1}$ is not allowed to penetrate $\Omega_{h 2}$ ). Conversely if we choose the second body as slave and the first one as master (in this case we have $n_{C 2}$ constraints ) we will not obtain exactly the same results, thus the user must choose the best slave/master. Otherwise there is some criterions to choose a body as slave, for example if it has the finest mesh or if is less stiffer than the second body... But what if this body has the finest mesh and is more stiffer than the second one. Therefore we conclude that there is a difficulty to choose the best slave/master, this issue also occurs in the case of self-contact or multi-body contact problems. Hence the need to create a symmetric formulation in order to not distinguish anymore between slave and master.

One simple way to create a symmetric formulation, is to take into account an additional non-penetration constraints by considering the second body as slave and the first one as master, in other word the problem becomes
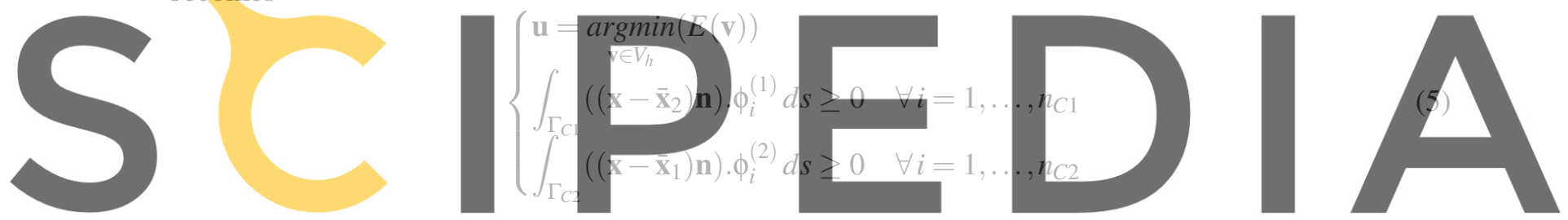

For linear elastic problems, the constraints in the problem (5) are linear, indeed the deformations are

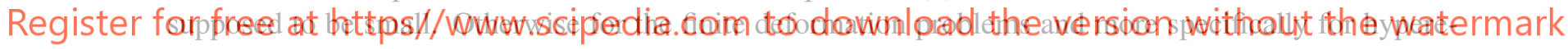
lastic materials, it is not the case, but we can transform the problem (5) into a sequence of minimization problems with linear constraints, using a fixed point algorithm. The gradient of the constraints in the problem (5) may be linear dependent, and thus generating a numerical difficulties, we will see next how to avoid these difficulties.

\section{Numerical optimization by the interior point method}

After the finite element discretization, the contact problem is transformed into a constrained optimization one, for this purpose the interior point method is used in order to solve it. Let $x \in \mathbb{R}^{n}$ denotes the degree of freedom of the displacement field $\mathbf{u}$ (using the finite element method), and $c: \mathbb{R}^{n} \rightarrow \mathbb{R}^{m}$ the linear constraints vector where $m=n_{C 1}+n_{C 2}$, the contact problem becomes

$$
\left\{\begin{array}{l}
\operatorname{Min}_{x \in \mathbb{R}^{n}} E(x) \quad \text { such that } \\
c(x) \geq 0
\end{array}\right.
$$


Note that the inequality between two vectors is the inequality between each component of these two vectors. Introducing the slack variables $s$, the problem (6) is equivalent to

$$
\left\{\begin{array}{l}
\underset{(x, s) \in \mathbb{R}^{n} \times \mathbb{R}^{m}}{\operatorname{Min}} E(x) \quad \text { such that } \\
c(x)-s=0 \\
s \geq 0
\end{array}\right.
$$

The constraints are linear, therefore the constraints are qualified for the KKT (Karush-Kuhn-Tucker) system, The first-order optimality or the KKT conditions for the problem (7) are the following

$$
\left\{\begin{array}{l}
\nabla_{x} E(x)+\nabla_{x} c(x) \lambda=0 \\
-\lambda-z=0 \\
c(x)-s=0 \\
S Z e=0 \\
s, z \geq 0
\end{array}\right.
$$

where $\lambda$ and $z$ are respectively the Lagrange multipliers of the equality constraints and the bound constraints, $S$ and $Z$ are two diagonal matrices where $S_{i i}=s_{i}$ and $Z_{i i}=z_{i}$, finally $e^{T}=(1, \ldots, 1)$. Applying a homotopy method to the problem (8) (see [5]) with the homotopy (also called barrier) parameter $\mu$.We obtain the following equations
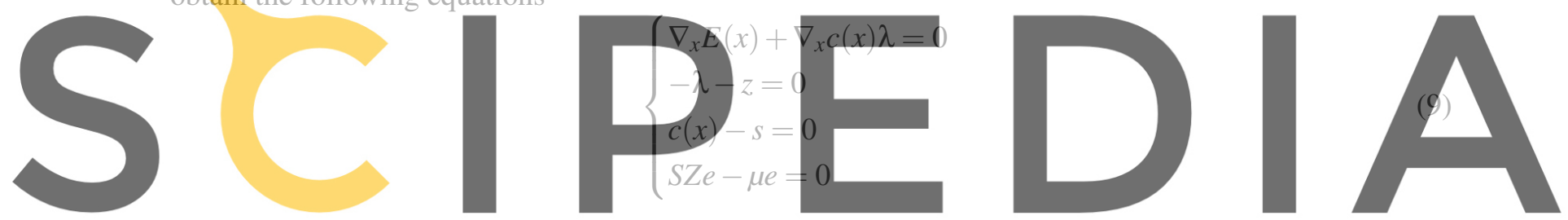

with $s, z \geq 0$ and $\mu$ converging to zero. Note if $\mu=0$ then the problem (9) becomes the KKT system of

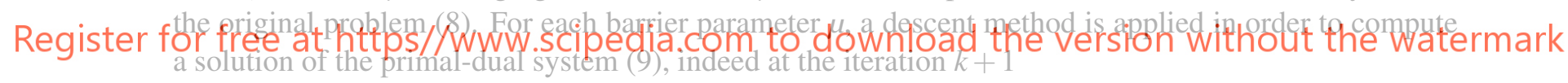

$$
\left\{\begin{array}{l}
x_{k+1}=x_{k}+\alpha_{k} d_{k}^{x} \\
s_{k+1}=s_{k}+\alpha_{k} d_{k}^{s} \\
\lambda_{k+1}=\lambda_{k}+\alpha_{k} d_{k}^{\lambda} \\
z_{k+1}=z_{k}+\alpha_{k}^{z} d_{k}^{z}
\end{array}\right.
$$

where $\alpha_{k}, \alpha_{k}^{z}$ are the step sizes, and $d_{k}^{x}, d_{k}^{s}, d_{k}^{\lambda}, d_{k}^{z}$ are respectively the descent directions of $x, s, \lambda$, $z$. Besides $s_{k+1} \geq 0, z_{k+1} \geq 0$ by using the fraction-to-the-boundary rule [5] to choose, $\alpha_{k}, \alpha_{k}^{z}$. In the following, a Newton's method is applied in order to compute the descent directions. By considering that our constraints are linear, we obtain the following linear system

$$
\left[\begin{array}{cccc}
\nabla_{x x} E\left(x_{k}\right) & 0 & \nabla_{x} c\left(x_{k}\right) & 0 \\
0 & 0 & -I & -I \\
\nabla_{x} c\left(x_{k}\right)^{T} & -I & 0 & 0 \\
0 & Z_{k} & 0 & S_{k}
\end{array}\right]\left(\begin{array}{c}
d_{k}^{x} \\
d_{k}^{s} \\
d_{k}^{\lambda} \\
d_{k}^{z}
\end{array}\right)=-\left(\begin{array}{c}
\nabla_{x} E\left(x_{k}\right)+\nabla_{x} c\left(x_{k}\right) \lambda_{k} \\
-\lambda_{k}-z_{k} \\
c\left(x_{k}\right)-s_{k} \\
S_{k} Z_{k} e-\mu e
\end{array}\right)
$$


This linear system (11) can be reduced into

$$
\left[\begin{array}{ccc}
\nabla_{x x} E\left(x_{k}\right) & 0 & \nabla_{x} c\left(x_{k}\right) \\
0 & S_{k}^{-1} Z_{k} & -I \\
\nabla_{x} c\left(x_{k}\right)^{T} & -I & 0
\end{array}\right]\left(\begin{array}{c}
d_{k}^{x} \\
d_{k}^{s} \\
d_{k}^{\lambda}
\end{array}\right)=-\left(\begin{array}{c}
\nabla_{x} E\left(x_{k}\right)+\nabla_{x} c\left(x_{k}\right) \lambda_{k} \\
-\mu S_{k}^{-1} e-\lambda_{k} \\
c\left(x_{k}\right)-s_{k}
\end{array}\right)
$$

with the additional equation $d_{k}^{z}=\mu S_{k}^{-1} e-z_{k}-S_{k}^{-1} Z_{k} d_{k}^{s}$. The matrix multiplied by the descent directions vector in the equation (12) has the form of

$$
K=\left[\begin{array}{cc}
G & A^{T} \\
A & 0
\end{array}\right]
$$

where $G=\left[\begin{array}{cc}\nabla_{x x} E\left(x_{k}\right) & 0 \\ 0 & S_{k}^{-1} Z_{k}\end{array}\right], A=\left[\nabla_{x} c\left(x_{k}\right)^{T} \quad-I\right]$.

Theorem 1. Suppose that the matrix $\nabla_{x x} E\left(x_{k}\right)$ is positive definite (for example a linear elastic material), suppose that $s_{k}>0$ then for a fixed $\mu$ the matrix $K$ is non singular.

Proof. One has to prove that $A$ is a full row rank matrix ( $\operatorname{rank}=m$ ), and $G$ is positive definite in the null space of $A$, otherwise speaking if $Z_{(n+m) \times n}$ is the full rank matrix where its columns are a basis for the null space of $A, Z^{T} G Z$ is positive definite, see [6].

First of all, the matrix $A$ is full row rank $(\operatorname{rank}=m)$, and the matrix $S_{k}^{-1} Z_{k}$ is positive, thanks to the fraction-to-the boundary rule. Let $X \in \mathbb{R}^{n}$, we want to prove that $Z^{T} G Z$ is positive definite. Indeed

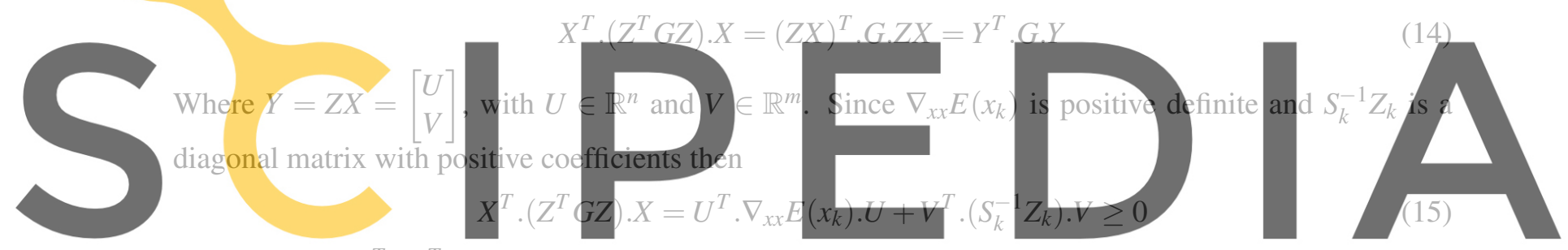

Suppose that $X^{T} \cdot\left(Z^{T} G Z\right) \cdot X=0$, thus form the equation (15) and from the positive definiteness of

Register for fre

$$
Y=Z X=\left[\begin{array}{l}
0 \\
V
\end{array}\right]
$$

By definition $Y$ belongs to the null space of $A$ then

$$
0=A . Y=\left[\begin{array}{ll}
\nabla_{x} c\left(x_{k}\right)^{T} & -I_{m \times m}
\end{array}\right]\left[\begin{array}{c}
0 \\
V
\end{array}\right]=-V
$$

Therefore $V=0$ and $Y=Z X=0$. Because $Z$ has full column rank then $X=0$. We deduce that the matrix $Z^{T} G Z$ is positive definite.

\subsection{Some technical discussions}

Considering the KKT system (9), we proved that the Lagrange multipliers solutions of this system are bounded for all barrier parameters $\mu$, in addition we showed that the slack variables $s_{\mu}$ also solutions of the KKT system (9), will not converge dramatically to zero, and are greater than a constant multiplied by $\mu$, otherwise speaking $\left\|s_{\mu}\right\|_{\infty} \geq m . \mu \quad \forall \mu \geq 0$. The proof is very similar to the one in the next section. In the following the vector norm $\|\cdot\|$ denotes the maximum vector norm $\|\cdot\|_{\infty}$. 


\subsubsection{Global convergence}

In order to solve the minimization problem with linear constraints (7), we will solve a sequence of barrier problems (9) with a decreasing barrier parameter $\mu$, converging to zero. Let $\varepsilon_{t o l}>0$ be a given error tolerance, and $K>0$ a given positive constant. For each barrier parameter $\mu$, let the error function $E_{\mu}$ be defined as:

$$
E_{\mu}:=\max \{\|\nabla E+\nabla c . \lambda\|,\|\lambda+z\|,\|c-s\|,\|S Z e-\mu e\|\}
$$

The algorithm to solve the minimization problem with linear constraints (7) is described briefly in the following box 1 .

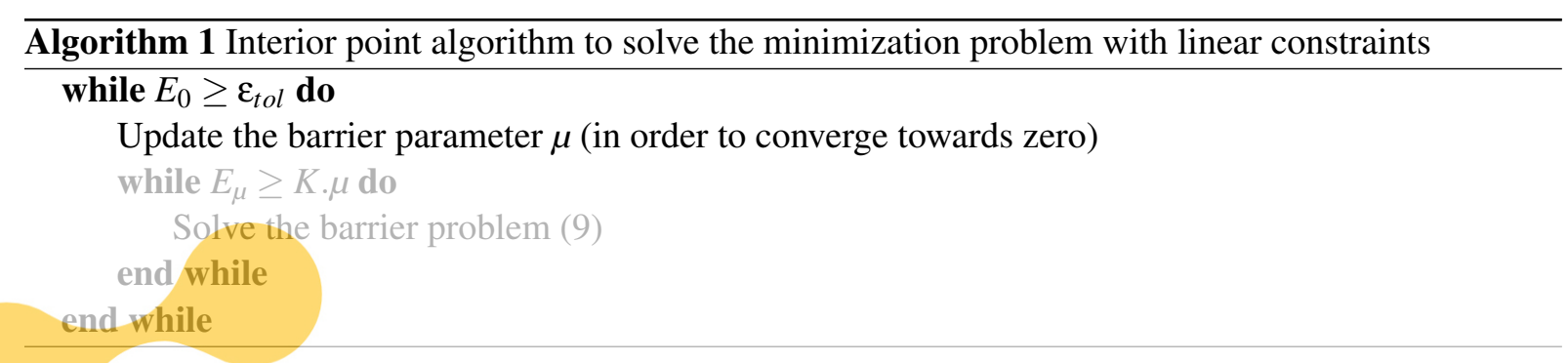

In the algorithm (1) and for each barrier parameter $\mu$, let $\left(x_{\mu}, s_{\mu}, \lambda_{\mu}, s_{\mu}\right)$ be a solution of each barrier problem, where the error $E_{\mu}$ is supposed to satisfy
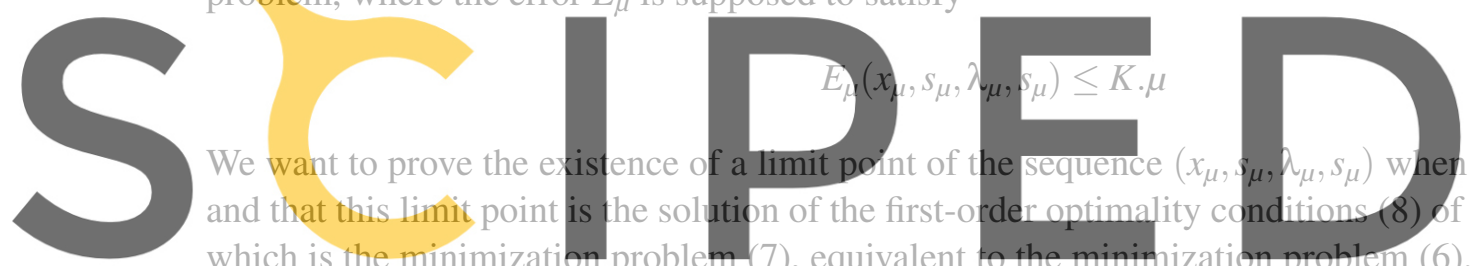

which this limit point is

, equivalen

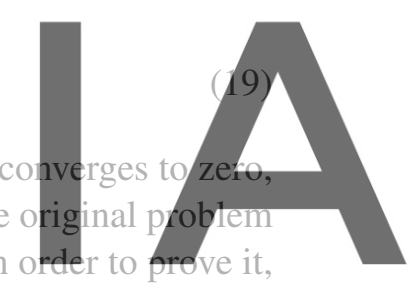

we will show that the sequence $\left(x_{\mu}, s_{\mu}, \lambda_{\mu}, s_{\mu}\right)$ is bounded.

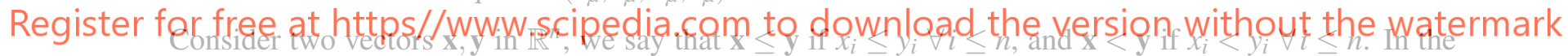

proof we will need a theorem of the alternative, due to Gordan [13], which states the following theorem.

Theorem 2. Consider a matrix $A_{n \times m}$. Exactly one of the following propositions has a solution:

1-There exists $x \in \mathbb{R}^{m}$ such that A. $x>0$

2-There exists $y \in \mathbb{R}^{n}$ such that $y^{T} . A=0, y \geq 0, y \neq 0$

In addition, we consider the following assumptions:

\section{Assumption 1.}

- The constraints vector $c: \mathbb{R}^{n} \rightarrow \mathbb{R}^{m}$ is linear

- There exists $\bar{x} \in \mathbb{R}^{n}$ such that $c(\bar{x})>0$

First $x_{\mu}$ is assumed to be bounded and thus belongs to a compact set $\Omega$. From the inequality (19) we have

$$
\left\|c\left(x_{\mu}\right)-s_{\mu}\right\| \leq K . \mu
$$

The application $c$ is continuous on the compact $\Omega$, thus from the inequality (20) we deduce that $s_{\mu}$ is bounded. 
In the algorithm $s_{\mu}$ and $z_{\mu}$ are chosen to be positive, thanks to the fraction-to-boundary rule. From the inequality (19) we obtain

$$
\lambda_{\mu} \leq K \mu-z_{\mu}
$$

Next we will be inspired by a proof which can be found in [7], by proving that the Lagrange multiplier $\lambda_{\mu}$ is bounded. Suppose that it is not the case and $\left\|\lambda_{\mu}\right\| \rightarrow+\infty$. As was mentioned, $z_{\mu} \geq 0$, we notice from the inequality (21) that if a component of $\lambda_{\mu}$ is positive then it is of the order of $K \mu$ and converges to zero. Therefore $\exists N>0$ such that $\forall n \geq N \Rightarrow e^{T} \cdot \lambda_{\mu_{n}} \leq 0$, moreover $d_{n}:=\frac{\lambda_{\mu_{n}}}{e^{T} . \lambda_{\mu_{n}}}$ is bounded. We deduce that there exists a subsequence of $d_{n}$ also denoted $d_{n}$ such that

$$
d_{n} \underset{n \rightarrow \infty}{\longrightarrow} d^{*} \text { with } d^{*} \geq 0
$$

Otherwise $e^{T} \cdot d^{*}=1$, thus $d^{*} \geq 0$ and $d^{*} \neq 0$.

From the error definition (18) and from the inequation (19) we have

$$
\left\|\frac{\nabla E\left(x_{\mu_{n}}\right)}{e^{T} \cdot \lambda_{\mu_{n}}}+\nabla c\left(x_{\mu_{n}}\right) \cdot \frac{\lambda_{\mu_{n}}}{e^{T} \cdot \lambda_{\mu_{n}}} \mid=\frac{1}{\left|e^{T} \cdot \lambda_{\mu_{n}}\right|}\right\| \nabla E\left(x_{\mu_{n}}\right)+\nabla c\left(x_{\mu_{n}}\right) \cdot \lambda_{\mu_{n}} \| \leq \frac{K \mu_{n}}{\left|e^{T} \cdot \lambda_{\mu_{n}}\right|}
$$

Thus if $n \rightarrow \infty$ then

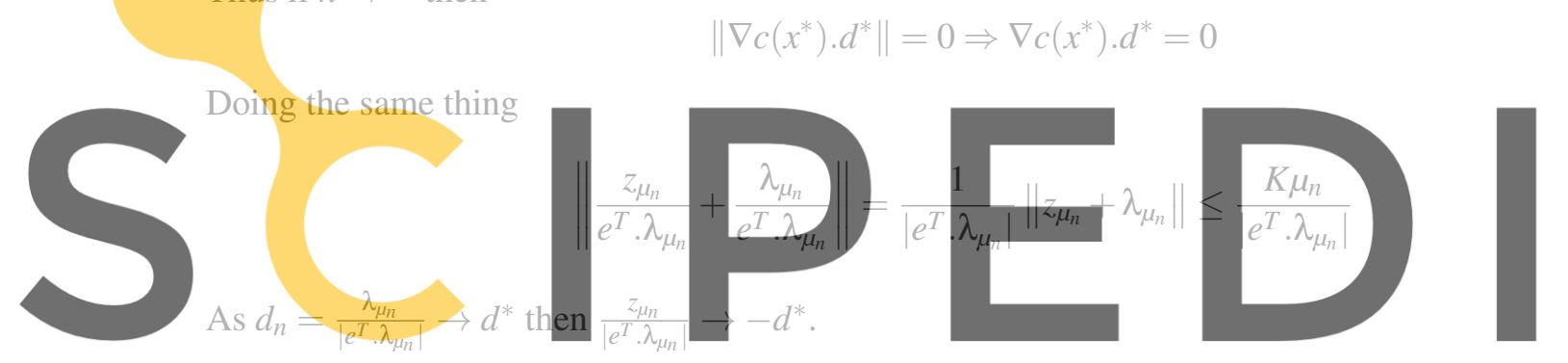

We have also from the error definition, that for each component $i$ Register for free at https//www.scipedia.com to download the version without the watermark $\left|s_{\mu_{n}}^{(i)} \cdot z_{\mu}^{(i)}-\mu\right| \leq K \cdot \mu$

If we multiply the equation (26) by $\frac{1}{\left|e^{T} \cdot \lambda_{\mu_{n}}\right|}$ and taking $n \rightarrow \infty$ we obtain

$$
s_{i}^{*} \cdot d_{i}^{*}=0 \quad \forall i=1, \ldots, m
$$

Let $I=\left\{i \mid s_{i}^{*}=0\right\}$, this set is not empty because if it is not the case, then using the fact that $d^{*} \neq 0$ we will have a contradiction with the equation (27). If we take a vector $v \in \mathbb{R}^{m}, v_{I}$ denotes the vector with the components $v_{i}$ where $i \in I$. We have $c_{I}\left(x^{*}\right)=s_{I}^{*}=0$ and from equation (27) if $i \notin I, d_{i}^{*}=0$. Thus

$$
e_{I}^{T} \cdot d_{I}^{*}=e^{T} \cdot d^{*}=1 \text { and } d_{I}^{*} \geq 0
$$

We conclude that

$$
d_{I}^{*} \neq 0 \text { and } d_{I}^{*} \geq 0
$$

Otherwise from the equation (24)

$$
\nabla c\left(x^{*}\right) d^{*}=\nabla c_{I}\left(x^{*}\right) d_{I}^{*}=0
$$


In conclusion we obtain

$$
\left\{\begin{array}{l}
\left(d_{I}^{*}\right)^{T}\left(\nabla c_{I}\left(x^{*}\right)\right)^{T}=0 \\
d_{I}^{*} \geq 0 \\
d_{I}^{*} \neq 0
\end{array}\right.
$$

By the theorem of the alternative, we deduce that there is no vector $p$ such that

$$
\left(\nabla c_{I}\left(x^{*}\right)\right)^{T} p>0
$$

We will prove next that we have a contradiction.

Otherwise, let $c_{I}^{(i)}$ a component of $c_{I}$, we know that $c_{I}^{(i)}$ is linear, thus

$$
c_{I}^{(i)}(\bar{x})-c_{I}^{(i)}\left(x^{*}\right)=\left(\nabla c_{I}^{(i)}\left(x^{*}\right)\right)^{T} \cdot\left(\bar{x}-x^{*}\right)
$$

We have $c_{I}^{(i)}\left(x^{*}\right)=0$, and by the assumption $(1) c_{I}^{(i)}(\bar{x})>0$. Therefore

$$
\left(\nabla c_{I}^{(i)}\left(x^{*}\right)\right)^{T} \cdot\left(\bar{x}-x^{*}\right)>0
$$

besides

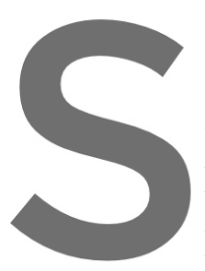

which contradicts the theorem of the alterngtive,

Lagrange multiplier $z_{\mu}$

In conclusion $y$ :

bounded, and
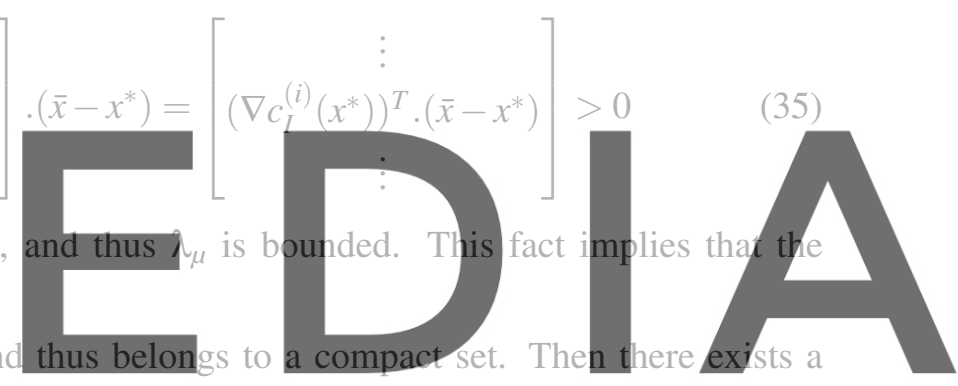

subsequence of $y_{\mu}$ converging to $y^{*}=\left(x^{*}, s^{*}, \lambda^{*}, z^{*}\right)$. Finally using the error definition and the inequality

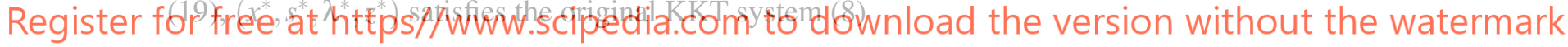

$$
\left\{\begin{array}{l}
\nabla E\left(x^{*}\right)+\nabla c\left(x^{*}\right) \lambda^{*}=0 \\
z^{*}=-\lambda^{*} \\
c\left(x^{*}\right)-s^{*}=0 \\
S^{*} Z^{*} e=0 \\
s^{*} \geq 0, z^{*} \geq 0
\end{array}\right.
$$

\section{Alternative formulations}

Another symmetric formulations were developed in order to solve the contact problems, more precisely the minimization of the energy with linear constraints. These formulations are a mixture of the penalty method and the interior point method. 


\subsection{First alternative formulation}

Instead of using the minimization problem (7), we will penalize the slack variables to be negative, and we obtain the following problem

$$
\left\{\begin{array}{l}
\underset{(u, s) \in \mathbb{R}^{n} \times \mathbb{R}^{m}}{\operatorname{Min}} E(u)+\sum_{i=1}^{m} \mu \cdot \eta\left(s_{i}\right) \quad \text { such that } \\
c(u)-s=0 \\
s \geq-\varepsilon
\end{array}\right.
$$

where $\mu$ the penalty factor $\left(\mu \rightarrow \infty\right.$ in this case), $\varepsilon>0$ and, $\eta: \mathbb{R} \rightarrow \mathbb{R}_{+}$a $C^{2}$ and a convex function with the following property: $\eta(x)>0 \forall x<0$ and $\eta(x)=0 \forall x \geq 0$. An example of a penalty function $\eta$

$$
\eta(x)=\left\{\begin{array}{l}
0 \text { if } x \geq 0 \\
-x^{3} \text { if }-1 \leq x<0 \\
3 x^{2}+3 x+1 \text { if } x \leq-1
\end{array}\right.
$$

In this formulation we ensure that the inequality in the problem (37) will not be activated. Note that the components values of the Hessian matrix due to the penalty terms are of the order $\mu . s_{i}$, thus near the solution these values are not high.
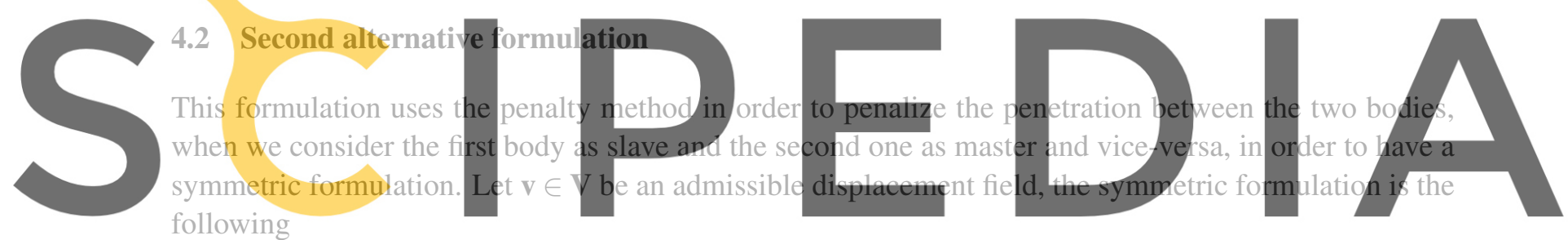
following

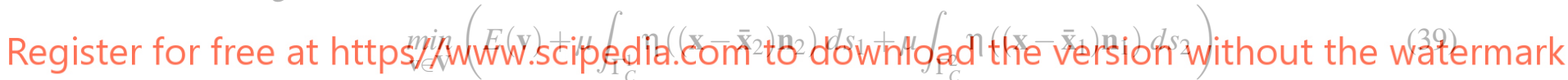

where $\mu$ the penalty factor, $\overline{\mathbf{x}}_{1}$ and $\overline{\mathbf{x}}_{2}$ are respectively the projection of the actual position $\mathbf{x}$ on the first body and the second body. $\mathbf{n}_{i}$ is the outward unit normal vector at $\overline{\mathbf{x}}_{i}$. Because the penalty terms are equal to zero when there is no contact, a bound on the displacement must be specified in the optimization process, and this bound is not obvious.

\section{Contact algorithm}

A fixed point method is used in order to transform the contact problem into a sequence of a minimization ones, with linear constraints, especially for the finite deformation problems. Indeed for the linear elastic problems, the constraints are linear by definition. More precisely for each iteration of the fixed point algorithm, the previous displacement is used in order to search for each integration point of the slave contact area, the closest segment (in 2D) or the closest triangle (in 3D) of the master body, and its position on this closest segment or triangle. The integration points have been employed, because the constraints are written in term of integrals. Finally the algorithm to solve the contact problems is briefly described in the following box (2). Note that the resolution of the linear system generated by the interior 
point method is solved using direct methods, for example the solver MUMPS (MUltifrontal Massively Parallel sparse direct Solver).

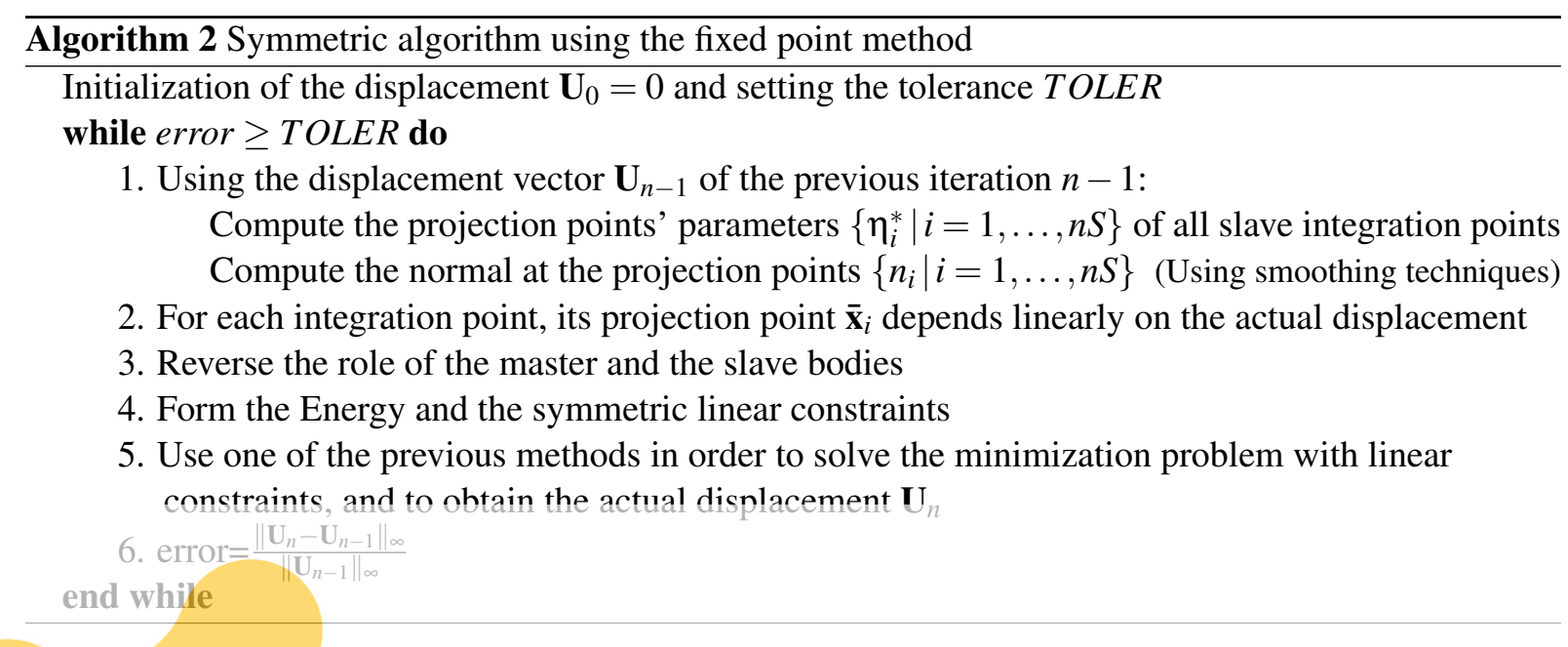

\section{Numerical examples}

The algorithm was developed using FreeFEM [1] and IPOPT [5] software, and validated against several test cases.
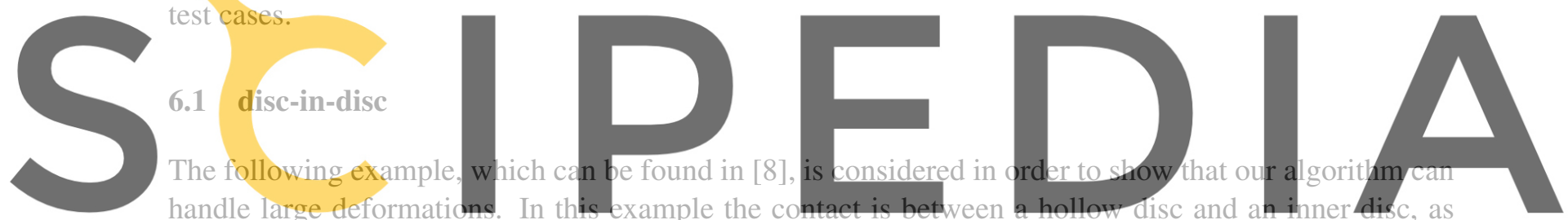

shown in the Figure (1), it is a quasi-static study where the inertia is not taken into account. The first

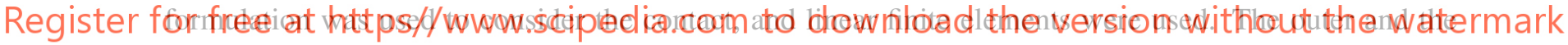
inner radius of the hollow disc are respectively $r_{h o}=2 . U L, r_{h i}=0.7 U L$, the radius of the inner disc is $r_{m}=0.6 U L$. Neo-Hookean material is considered for the two discs, with the following properties $E_{h}=1000 \cdot \frac{U F}{U L^{2}}, v_{h}=0$ for the hollow disc, and $E_{i}=2000 \cdot \frac{U F}{U L^{2}}, v_{i}=0.3$ for the inner disc, note that $U F$, $U L$ denote respectively the force and the length units. We impose a vertical downward displacement at all nodes of the inner disc, the outside of the hollow disc is fixed. The maximal displacement imposed is $1.125 U L$ and is done by 100 load increments. The deformation states of the two discs at the steps 0,50 , 100 are depicted in the following figure (Figure (1)).

\subsection{Multi-body contact between three beams}

In this example we consider the bending of three cantilever beams as shown in the Figure (2), the goal of this example is to show that the contact algorithm can work in three-dimensional space and can handle multi-body contact problems. The first formulation was used to consider the contact, and linear finite elements were used. The dimension of the three beams are the same, indeed the length of each beam is 200. $U L$ and each cross section is a square of dimension 25.UL. The three beams have the same elastic 

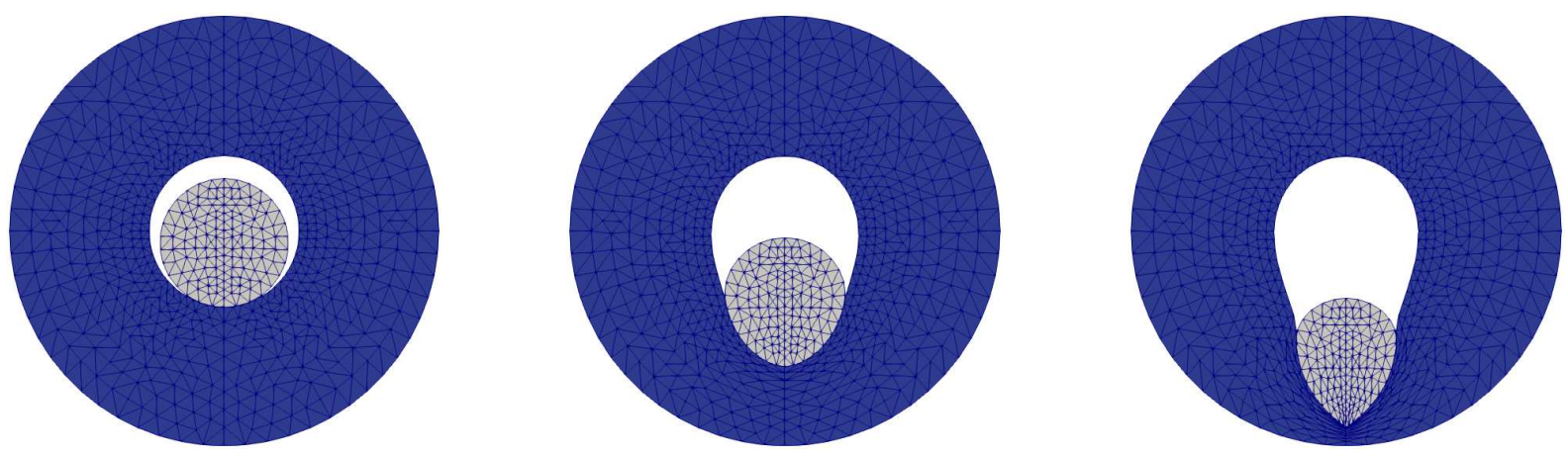

Figure 1: The deformation states at the steps $0,50,100$

material properties $E=2.10^{5} \frac{U F}{U L^{2}}, v=0$. We apply a downward vertical surface load of $f=100 \frac{U F}{U L^{2}}$ at the upper surface of the top beam. We can see in the Figure (2) the deformations and the sliding between the beams.
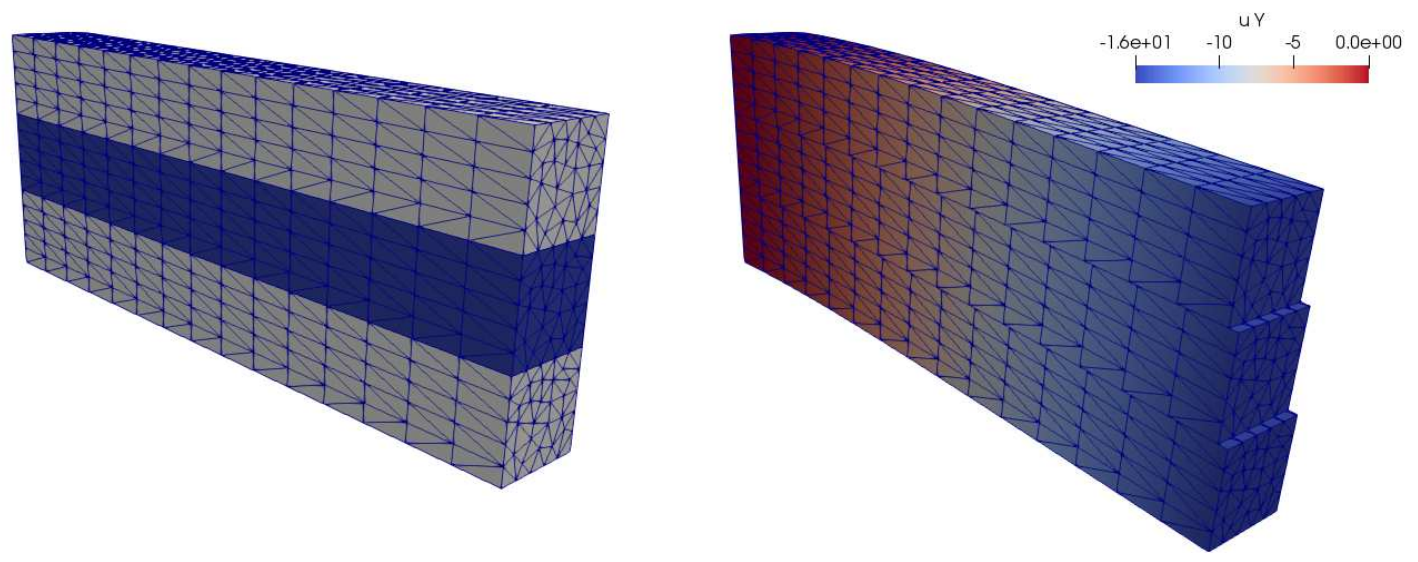

Figure 2: The sliding between beams. The initial configuration (on the left), the deformations and the sliding (on the right)

\section{CONCLUSIONS}

We developed an algorithm to solve frictionless contact problems, using FreeFEM and IPOPT software. The contact problem is written in the form of a sequence of minimization problems with linear constraints, and each minimization problem is solved using the interior point method. The advantages of this algorithm is its simplicity, in addition the algorithm is symmetric, therefore the user does not distinguish anymore between the slave and the master body, which is very useful for the self-contact and multi-body contact problems. 


\section{REFERENCES}

[1] Hecht, F. New development in FreeFem++. J. Numer. Math. (2012) 20(3-4):251-265.

[2] Houssein, H. and Garnotel, S. and Hecht, F. Frictionless contact problem for hyperelastic materials with interior point optimizer. (2019)

[3] Belgacem, F Ben. and Hild, P. and Laborde, P. The mortar finite element method for contact problems. Mathematical and Computer Modelling (1998) 28(4-8):263-271.

[4] Hild, Patrick. Problèmes de contact unilatéral et maillages éléments finis incompatibles. PhD thesis, (1998).

[5] Wächter, Andreas. and Biegler, Lorenz T. On the implementation of an interior-point filter line-search algorithm for large-scale nonlinear programming. Mathematical programming (2006) 106(1):25-57.

[6] Nocedal, Jorge. and Wright, Stephen. Numerical optimization. Springer Science \& Business Media, (2006).

[7] Anstreicher, Kurt M. and Vial, Jean-Philippe. On the convergence of an infeasible primaldual interior-point method for convex programming. Optimization methods and software (1994) 3(4):273-283.

[8] Wriggers, Peter. Computational Contact Mechanics, Second Edition. Springer-Verlag, (2006).

[9] Kikuchi, N. and Oden, JT. Contact problems in elasticity: a study of variational inequalities and finite element methods. siam, (1988) 8.

[10] Tanoh, G. and Renard, Y. and Noll, D. Computational experience with an interior point algorithm for large scale contact problems. Optimization Online (2004) 10(2).

[11] Temizer, I. and Abdalla, MM. and Gürdal, Z. An interior point method for isogeometric contact. Computer Methods in Applied Mechanics and Engineering (2014) 276:589-611.

[12] Kučera, Radek. and Machalová, Jitka. and Netuka, Horymír. and Ženčák, Pavel. An interior-point algorithm for the minimization arising from 3D contact problems with friction. Optimization methods and software (2013) 28(6):1195-1217.

[13] Perng, Cherng-tiao. On a class of theorems equivalent to Farkas's lemma. Applied Mathematical Sciences (2017) 11(44):2175-2184. 